\title{
Effect of reaction parameters in synthesis, characterisation of electrodeposited zinc nanohexagons
}

\author{
R. Singaravelan $\cdot$ S. Bangaru Sudarsan Alwar
}

Received: 12 May 2014/ Accepted: 15 July 2014/Published online: 27 August 2014

(C) The Author(s) 2014. This article is published with open access at Springerlink.com

\begin{abstract}
Nanocrystalline zinc was electrodeposited by reducing the $\mathrm{Zn}^{2+}$ ion from acid sulphate bath at room temperature. The effect of reaction parameters on the particle size, nature and spectral characteristics are deliberated. The surface morphology and texture of zinc nanoparticles (Zn NPs) were characterised by high resolution scanning electron microscopy (HR-SEM) and high resolution transmission electron microscopy (HR-TEM). SEM and TEM images showed the Zn NPs were in hexagonal structural morphology. Energy dispersive X-ray analysis reveals the chemical stoichiometry, and purity of the particles formed. The powder X-ray diffraction data indicates that the reflections of Zn NPs correspond hexagonal close packing structure with space group of $\mathrm{P} 63 / \mathrm{mmc}$. The line broadening was analysed by Debye-Scherrer equation and the average crystallite size of the zinc Nps synthesised at room temperature was in the range of $29 \mathrm{~nm}$. WilliamsonHall analysis was used to study the contribution of crystallite size and lattice strain on the peak broadening. The surface plasmon resonance peak for the Zn NPs was observed near the blue shift with optical band gap of $3.38 \mathrm{eV}$. The influences of the current density, $\mathrm{pH}$, additives, and concentration medium were analysed.
\end{abstract}

Keywords Electrodeposition $\cdot \mathrm{Zn}$ NPs $\cdot$ XRD . HR-SEM · UV-Visible · pH effect · TEM

R. Singaravelan $(\bowtie) \cdot$ S. B. S. Alwar

Post Graduate and Research Department of Chemistry,

D. G. Vaishnav College, Chennai, India

e-mail: shivramki31@gmail.com

\section{Introduction}

Nanomaterials have received a significant attention over the past few decades due to their distinguished performance and potential applications. Unique characteristics at nanodimension makes them suitable for multiple applications such as bio sensing [1, 2], photovoltaic, medicine [3], catalysis etc. Among these, metals with ultra-fine crystallite size with structural radius $<100 \mathrm{~nm}$ showed considerable changes in mechanical, physical, and chemical properties in comparison with the bulk materials. Metal nanoparticles are particularly interesting nanoscale systems because of the ease with which they can be synthesised and modified chemically. Control of dimension and morphology of the metal nanoparticles have aroused the interest of researchers in the design of functional devices due to the optical and electronic properties of nanometre-sized materials, which determine their applications, and can be adapted by varying their size and shape. So the synthesis and investigation of properties of these nano materials have become the most important research hotspot for the past few decades. Among the various metal nanoparticles, zinc nanoparticles $(\mathrm{Zn}$ NPs) have been widely investigated because of the unusual properties due to their size and shape, which find wide range of technical applications. Zn NPs have received a considerable amount of attention for their applications in super hydrophobic substrate [4], rechargeable electrical batteries [5], gas sensor [6], corrosion resistance materials [7-9], and as substrates in the studies of catalysis [10].

In general, it is difficult to synthesis nanoparticles of reactive metal like $\mathrm{Zn}$ from electro-chemical method directly. Since metals are deposited electrolytically from solutions in the increasing order of their reduction potential (inverse order of their position) in the electrochemical series, and since hydrogen is positioned under zinc, it 
should, essentially, be depleted from a solution before any zinc to be deposited. Though there is a possibility for both hydrogen and zinc to be deposited simultaneously, zinc alone is deposited since hydrogen has an overvoltage on zinc. However, due to hydrogen overvoltage, hydrogen becomes less noble than zinc, and this distinctiveness makes it possible for the deposition of electrolytically less noble metal like zinc. It often results in unique growth of particles due to various reaction parameters used for electrochemical method. Zinc has high hydrogen over potential depending somewhat on $\mathrm{pH}$. This kinetically hinders hydrogen evolution reaction and renders zinc fairly stable in aqueous solution. This fact makes it useful material for negative electrode in batteries, corrosion protection of steel, and so on. Also because of the high hydrogen over potential, the zinc ion can be reduced to metal, permitting quantitative determination of zinc by cathodic deposition.

The effects of additive agents and their influence on the surface morphology of the Zn NPs and on hydrogen overvoltage on cathode were also studied. The presence of additives has been shown to influence physical and mechanical properties of electrodeposits such as grain size, brightness, internal stress, pitting and even chemical composition. With increased acidity, the deposit becomes smoother, but does not adhere any better to the cathodic surface on which it is deposited.

The development of new-fangled methods for synthesis of metal Nps has been one of the interesting areas of research in the field of nanoscience. Of the various physical and chemical methods, radiation chemistry, laser ablation technique [11], thermal evaporation [12-14], biosynthesis $[15,16]$, and electrodeposition [17-21] are often used to synthesise zinc nanostructures. Of all the methodologies developed for the preparation of $\mathrm{Zn} \mathrm{NPs,} \mathrm{the} \mathrm{electro-}$ chemical method (bottom-up approach) offers a simple alternative mean for high yield of nanoparticles. This technique offers a simple method for size and shape controlled synthesis of metal nanoparticles.

Zinc can be electrodeposited from acid or alkaline [23], cyanide, non-cyanide [22], and bromide, sulphate [21], or chloride [24, 25] type of electrolyte. The sulphate type baths are used for the high-speed deposition and chloride baths are used for higher throwing power. Among the acidic media, sulphate solution is used for electro deposition. Zinc sulphate is the most common acidic bath while chloride and fluoro-borate-based media are used less frequently. In the present electrochemical work, a simple, cost-effective, and efficient method is proposed for the synthesis of Zn Nps. In comparison with all the above methods, electrodeposition technique can be used for the large-scale nanoparticle synthesis. The effect of an electrolyte concentration on the crystal morphology is also studied by varying the concentration.
The processing conditions, such as bath temperature, $\mathrm{pH}$, current density, additives, and bath composition, have a profound effect on the properties of zinc electrodeposits such as grain size, surface morphology, and preferred orientation. In general, interrelated parameters such as current density, electrode potential difference, bath temperature, metal ion concentration, electrolyte conductivity, bath additives, electrode material, and geometry, all contribute to the nature and character of the deposition.

Many characterisation and analytical techniques have been applied to the study of nanomaterial and nanostructures, only a few widely used methods are reviewed in this paper. Structure morphology characterisations were carried out using high resolution scanning electron microscopy (HR-SEM), high resolution transmission electron microscopy (HR-TEM), Ultraviolet-Visible spectrum, energy dispersive X-ray analysis (EDAX), and powder X-ray diffraction (XRD), an excellent correlation was found between particle sizes determined from theoretical and experimental data.

\section{Experimental}

Materials and method

$\mathrm{ZnSO}_{4} \cdot 7 \mathrm{H}_{2} \mathrm{O}(99.5 \%$ pure), hydrazine sulphate, and CTAB of AR grade were purchased from M-merck, and used as received without further purification. The twoelectrode cell was used for the electrodeposition process. The cathode used was electrolytic grade zinc sheet $20 \times 30 \mathrm{~mm}^{2}$, and the glassy carbon electrode was used as a counter electrode in our electrochemical cell. Prior to the electrolysis, both the electrodes were mechanically polished and washed with acetone followed by double distilled water. Both the electrodes were immersed in the electrolyte, and are held parallel to each other at the distance of $3 \mathrm{~cm}$. During electrolysis the electrolyte was frequently agitated to allow the gases to escape and permit the zinc to deposit on the cathode surface.

Before the electrolysis, the $\mathrm{pH}$ of the electrolyte was also varied between 2.8 and 3.2 using hydrazine sulphate buffer. A controlled-current electrolysis was used throughout for a typical current of $10 \mathrm{~mA}$, where the constant current was drawn from the direct power supply (regulated DC power supply). It was seen that the size of the nanoparticles decreased with the decrease in the electrolyte concentration because of the high applied voltage. The temperature was controlled at $35^{\circ} \mathrm{C}$. At the end of the reaction, the cathode with zinc deposit was repeatedly washed with distilled water and dried under vacuum, and carefully scratched from the cathode surface. The $\mathrm{pH}$ of the zinc sulphate electrolyte was recorded before and after 
electrolysis and found to be decreasing due to decrease in the concentration.

\section{Synthesis of Zn NPs}

The most speckled and versatile preparation route appears to be the so-called "sacrificial anode", electrolysis that is usually carried out in the presence of the nanoparticle stabilisers. The Zn Nps prepared in this method are present at zero oxidation state, which seems to be a major problem with the wet chemical methods involved in the zinc nanoparticle synthesis. Electrodeposited Zn NPs has a matte dark grey appearance. This provides a platform for new metallic nanoparticles synthesis. As a fact, $\mathrm{Zn}$ is more reactive, and reduction of $\mathrm{Zn}^{2+}$ ions are found to be a slow reaction and longer time is required for the Zn NPs deposition in comparison with the electrodeposition of other metal nanoparticles.

Several mechanisms have been proposed for the overall cathodic reduction of $\mathrm{Zn}^{2+}$ ions. The charge transfer might occur from the bivalent $\mathrm{Zn}^{2+}$ ions in the electrolyte in two successive mono-electronic steps involving mono-valent $\mathrm{Zn}^{2+}$ ion intermediate. There is a little evidence that, zinc forms a univalent ion in solution, although it is postulated as an intermediate for reaction scheme. Evidence in radiation studies indicates a transitory stability of univalent zinc of about $t_{1 / 2}=10^{-6} \mathrm{~s}$ in aqueous media. In aqueous solution zinc seldom exist as the single ion and most probably exists as the tetra-coordinate aqueous ion.

$\mathrm{Zn}^{2+}+\mathrm{e}^{-} \rightarrow \mathrm{Zn}^{+}$

$\mathrm{Zn}^{+}+\mathrm{e}^{-} \rightarrow \mathrm{Zn}$

At the end of electrolysis, the deposited Zn NPs were carefully removed from the cathode surface washed with distilled water, dried, and weighed. During electrolytic process any current causing changes are also considered. The study of electrode reactions is unique in the sense that within limits the rate of the reaction can be controlled by simply increasing or decreasing the current throughout the cell. According to Faraday's law of electrolysis, the overall chemical change produced by any given quantity of electricity can be accounted exactly. The current efficiency (CE) as the ratio between the actual metal deposited (or dissolved), $M_{\mathrm{a}}$, and that calculated from Faraday's law, $M_{\mathrm{t}}$, in percentage is given by the equation.

$\mathrm{CE}=\frac{M_{\mathrm{a}}}{M_{\mathrm{t}}} \times 100$

And the cathodic current efficiency (CCE) for Zn NPs calculated as

$\mathrm{CCE}=\frac{Q_{\mathrm{Zn}}}{Q_{\text {total }}} \times 100$, where $Q_{\mathrm{Zn}}$ is the real charge estimated from the quantity of zinc deposited and $Q_{\text {total }}$ is the theoretical charge calculated from Faraday's law.

\section{Effect of reaction parameters}

\section{Effect of $\mathrm{pH}$}

The $\mathrm{pH}$ of the bath has remarkable effect on the nature and size of the Zn NPs. Zinc forms basic zincate complex with hydroxide ion at higher $\mathrm{pH}$ and it forms zinc ions at low $\mathrm{pH}$ (below 3.0) conditions, which indicate the amphoteric nature of zinc in solution. It was also observed that, at high $\mathrm{pH}$ conditions the $\mathrm{Zn}$ deposit was spongy or porous, whereas at low $\mathrm{pH}$ ranging 3.2-3.6, the $\mathrm{Zn}$ NPs showed a hexagonal close packing (HCP) structure. The optimum acidity for the electrolyte lies between 3.2 and 3.6, and it was adjusted with hydrazine sulphate buffer solution. In addition, hydrogen evolution and a consequent decrease in current efficiency were observed. The optimum $\mathrm{pH}$ for the $\mathrm{Zn}$ electrodeposition was found to be 3.2 .

\section{Effect of current density}

The results show that the cathode current efficiency increases with current density and then decreases with further increase in current density. This is due to the hydrogen evolution occurring at the cathode along with zinc deposition at the high current densities.

\section{Effect of additives}

To improve the quality of the deposit, cetyl trimethyl ammonium bromide (CTAB) is added as a surfactant to the electrolytic bath in millimolar concentrations. Increasing the concentration of CTAB has a strong influence on the cathodic hydrogen over potential and results in decrease of hydrogen over potential. And it also causes hydrogen evolution rather than zinc deposition and results in the decrease in the current efficiency of the cell.

Hydrazine sulphate has been used as a strong reducing agent and has widely been used for preventing corrosion. The presence of hydrazine sulphate in the electrolytic solution was found to decrease the $\mathrm{pH}$ of the solution. In addition, the hydrazine binds dissolved oxygen in the electrolytic bath through the following reaction. It also reduces the metal oxide present in the electrolyte.

$\mathrm{N}_{2} \mathrm{H}_{6} \mathrm{SO}_{4}+\mathrm{O}_{2} \rightarrow \mathrm{N}_{2} \uparrow+2 \mathrm{H}_{2} \mathrm{O}+\mathrm{H}_{2} \mathrm{SO}_{4}$

$2 \mathrm{ZnO}+\mathrm{N}_{2} \mathrm{H}_{4} \rightarrow 2 \mathrm{Zn}+2 \mathrm{H}_{2} \mathrm{O}+\mathrm{N}_{2} \uparrow$

With increased acidity, the deposit becomes smoother, but does not adhere better to the zinc cathode surface on which it was deposited. The presence of CTAB gives a much smoother deposit, but does not particularly increase the adherence to zinc. 
Influence of electrolyte

Bath composition has profound effect on the surface morphology of the nanoparticles. When the concentration of the zinc ion increases, the particle size also increases as the $\mathrm{Zn}^{2+}$ ion population in the solution increases. By changing the composition of the electrolyte, it was found that the nanoparticles can be single crystal or polycrystalline. The morphology of the deposits and crystallographic orientation (texture) strongly depend on the electrolytic concentration. The texture is also found to depend on deposition time. Well-defined geometric shapes such as hexagon with clear sharp edges were obtained, when low concentration of the electrolyte is used. Various concentrations of electrolytes were used to obtain effective deposition. The optimised concentration of the electrolyte for the finest crystalline $\mathrm{Zn} \mathrm{NPs}$ is found to be $0.02 \mathrm{M}$. Agglomeration is observed when a higher concentration $<0.02 \mathrm{mmol}$ of the electrolytes is used.
Fig. 1 HR-SEM micrographs of electrodeposited zinc nanohexagons at a $\mathrm{pH} 4.2$ with $0.04 \mathrm{mM}$ concentration of the electrolyte without additives, b $\mathrm{pH} 3.6$ with $0.02 \mathrm{mM}$ concentration of the electrolyte with hydrazine, $\mathbf{c} \mathrm{pH} 3.2$ with $0.02 \mathrm{mM}$ concentration of the electrolyte with additives, $\mathbf{d ~} \mathrm{pH}$ 3.6 with $0.04 \mathrm{mM}$ concentration of the electrolyte with additives, e $\mathrm{pH} 3.2$ with $0.02 \mathrm{mM}$ concentration of the electrolyte with hydrazine, $\mathbf{f} \mathrm{pH} 3.2$ with $0.02 \mathrm{mM}$ concentration of the electrolyte with $\mathrm{CTAB}$ only
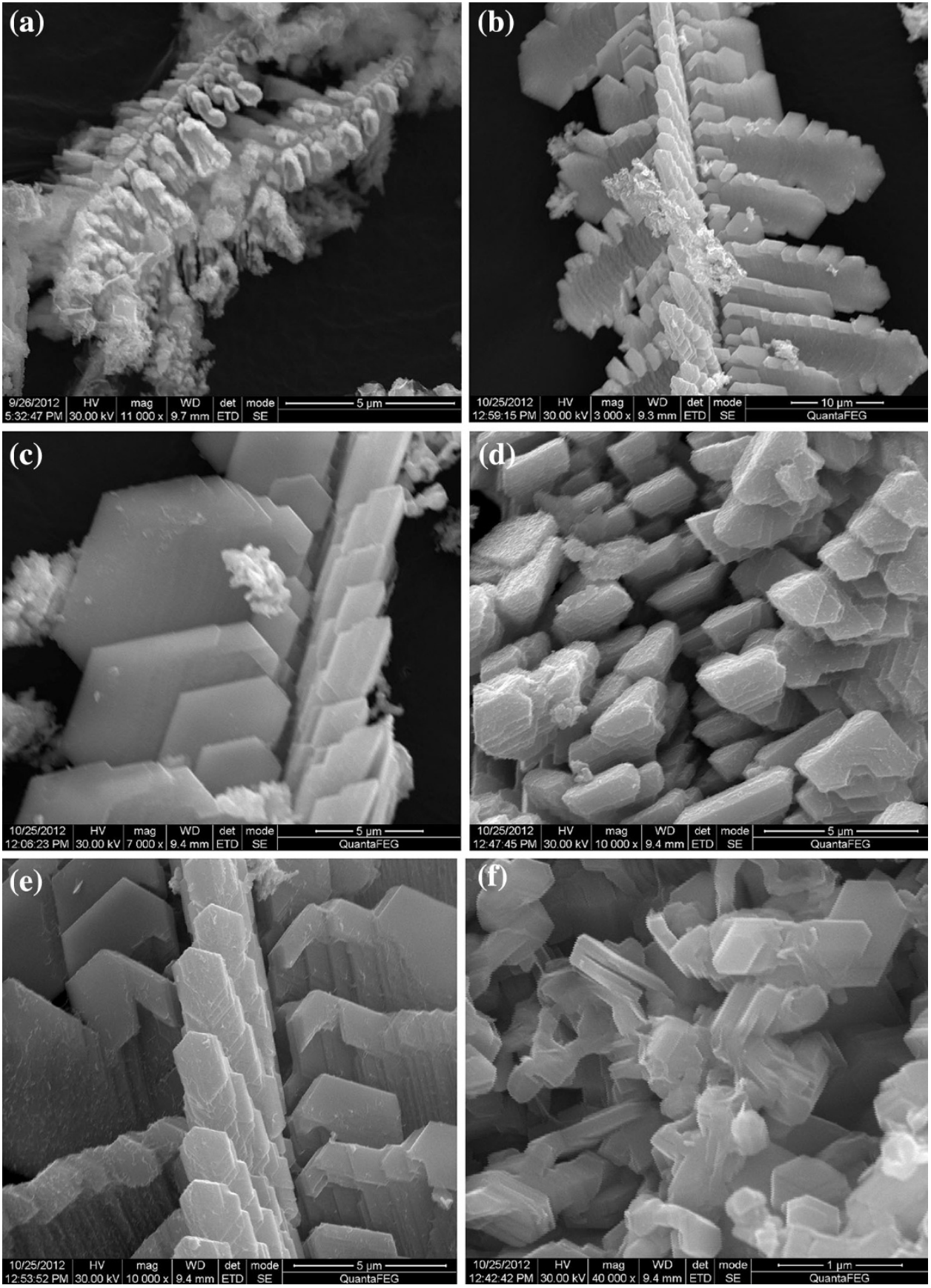


\section{Results and discussions}

\section{HR-SEM/EDAX}

The detailed structural characterisation was done by HRSEM. Surface morphology of Zn NPs was characterised by HR-SEM (FEI Quanta FEG 200). SEM images of the Zn NPs obtained from sulphate bath revealed valuable information regarding the purity of a nanoparticle sample as well as an insight on their degree of aggregation. From the SEM images, it is seen that the crystalline morphology was pure in composition. Furthermore, the SEM micrographs clearly show the high degree of dispersion and uniformity of Zn NPs. HR-SEM images of Zn NPs obtained with and without additives at optimised conditions of the electrolyte are shown in Fig. 1.

The quantitative chemical stoichiometry of $\mathrm{Zn}$ nanohexagons were investigated by energy dispersive X-ray spectroscopy (HITACHI, and S-2400), which affirmed the existence of an atomic zinc. Figure 2 illustrated the chemical nature of electrodeposited Zn NPs. From the peak, it can be understood that the zinc intensity is rich and the intensity of oxygen is almost zero. Hence, it is understood that the Zn NPs are not oxidised.

\section{HR-TEM}

TEM has been found to be a key method for the characterisation of various types of nanomaterial in the size

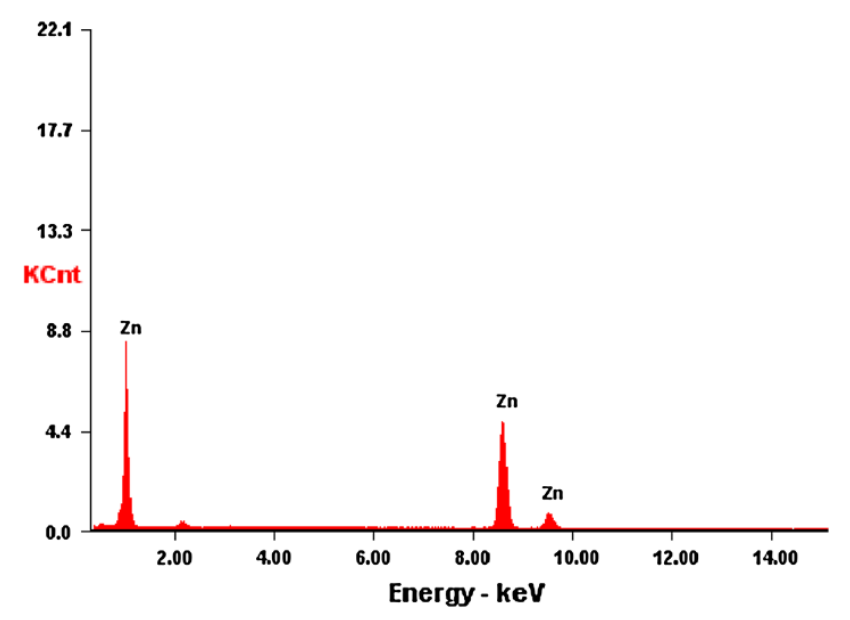

\begin{tabular}{|l|l|l|}
\hline Element & Wt\% & At\% \\
\hline ZnK & 100.00 & 100.00 \\
\hline Matrix & Correction & ZAF \\
\hline \hline
\end{tabular}

Fig. 2 The chemical stoichiometry of the $\mathrm{Zn}$ nanohexagons ranging from $10 \mathrm{~nm}$ to $1 \mu \mathrm{m}$. Figure 3 , shows a bright-field low magnification HR-TEM image of dendrite $\mathrm{Zn}$ nanohexagons, deposited under different concentrations. It is also seen that the dendrite is composed of layered nanohexagons.

\section{UV-Visible spectroscopy}

Metal nanoparticles resembles with those of bulk materials in their optical properties even in very small dimensions. When these materials are irradiated with suitable light energy electrons are excited and move from valence band to conduction band. Optical properties of the Zn NPs were characterised by UV-Visible spectroscopy and the surface plasmon resonance peak is seen in Fig. 4. It is very interesting to investigate the optical properties of $\mathrm{Zn}$ nanocrystals since it absorbs strongly in the visible region due to surface plasmon resonance. The absorption spectrum for the $\mathrm{Zn} \mathrm{NPs} \mathrm{at} \mathrm{different} \mathrm{reaction} \mathrm{conditions} \mathrm{is} \mathrm{also} \mathrm{shown} \mathrm{in}$ Fig. 4. It is also observed that the surface plasmon resonance for the Zn NPs is dependent on the reaction condition. From figure, it is evident that the $\mathrm{Zn}$ nanocrystals showed high absorbance in the wavelength (blue shift) range of $366 \mathrm{~nm}$ in the absorbance spectrum. The energy band gap of these Zn NPs was estimated using the relation,

$E_{\mathrm{g}}=\frac{h c}{\lambda}$

where $E_{\mathrm{g}}$ is the band gap, $h$ is the plank's constant, $c$ is the velocity of light and $\lambda$ is the maximum absorption wavelength. The band gap energy for the $\mathrm{Zn}$ Nps synthesised at optimum condition is $3.38 \mathrm{eV}$ and the band gap energies for the $\mathrm{Zn}$ Nps synthesised at various reaction conditions are shown in Table 1. This can be demonstrated by showing that nano materials have discrete quantized energy levels due to quantum confinement effects and consequently a larger band gap compared to that of bulk solids. The smaller the band gap, the more electrically conductive a material will be.

X-ray powder diffraction analysis

$\mathrm{X}$-ray diffraction (XRD) is the most useful technique used to characterise the crystallographic structure, crystalline size and preferred orientation in polycrystalline sample. XRD data were recorded using Seifert analyser with $\mathrm{Cu} \mathrm{k} \alpha$ $(\lambda=1.5406 \AA)$ as incident radiation and intensity data collected over a $2 \theta$ range of $10^{\circ}-70^{\circ}$. As can be seen in Fig. 5, there are four peaks at Bragg's angles of 36.31, $38.97,43.23$, and 54.33, respectively. These peaks correspond to the $002,100,101$, and 102 indicating the formation of $\mathrm{Zn} \mathrm{Nps.} \mathrm{The} \mathrm{diffraction} \mathrm{peaks} \mathrm{were} \mathrm{perfectly}$ indexed to the XRD pattern of pure zinc nano crystal 
Fig. 3 TEM micrographs of $\mathrm{Zn}$ nanohexagons synthesised at a $\mathrm{pH} 4.2$ and $0.04 \mathrm{mM}$ concentration, with $\mathrm{CTAB}$, b $\mathrm{pH} 3.2$ and $0.02 \mathrm{mM}$ concentration, with $\mathrm{CTAB}, \mathbf{c} \mathrm{pH}$ 3.6 and $0.01 \mathrm{mM}$ concentration, without CTAB, d pH 3.2 and $0.02 \mathrm{mM}$ concentration, without CTAB
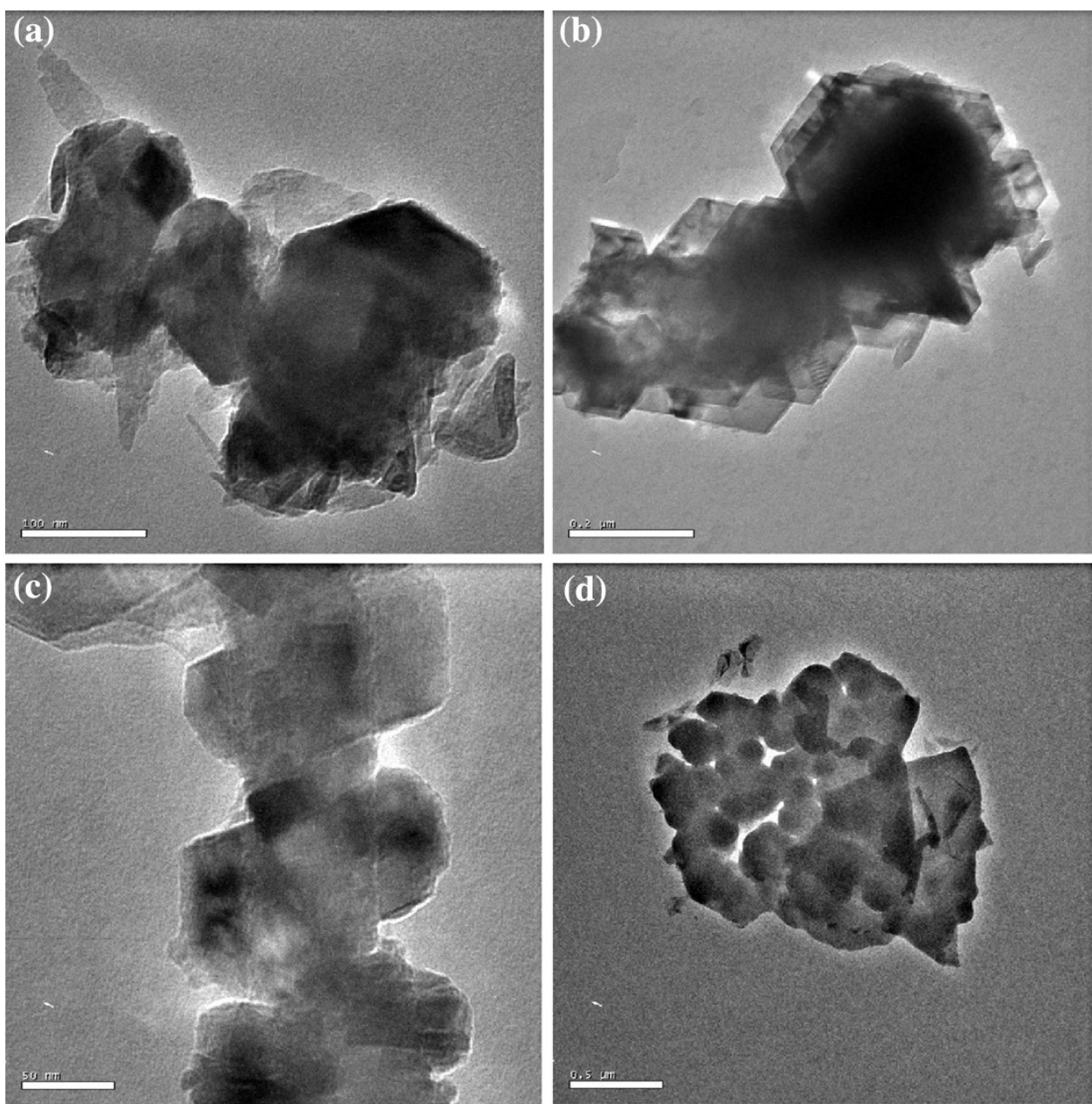

(JCPDS file no. 04-0831) with hexagonal close-packed arrangement, which belongs to the space group P63/mmc. $\mathrm{X}$-ray diffraction pattern contains an intense peak at $2 \theta=43.23^{\circ}$ (101), which is the characteristic of zinc phase and it was present almost in all deposits with significant intensity. The diffraction patterns for the $\mathrm{Zn} \mathrm{Nps}$ synthesised at different reaction parameters are also shown in Fig. 5. XRD pattern indicating that the Zn Nps synthesised by this method exhibit first-rate crystallinity and a high purity.

\section{Scherrer method}

$\mathrm{X}$-Ray peak broadening was used to estimate the grain size with the help of Scherrer formula. The crystalline size of the Zn NPs was determined by line broadening method using Debye-Scherrer equation (Scherrer 1918) and the average crystalline size for the Zn NPs synthesised under optimum condition was found to be $29 \mathrm{~nm}$.

$D=\frac{K \lambda}{\beta_{h k l} \cos \theta}$ where $D$ is the grain size $(\mathrm{nm}), \lambda$ is the wavelength of $\mathrm{X}$-ray used $(\mathrm{nm}), \theta$ is the diffraction angle $\left(^{\circ}\right), \beta$ is the full width half maximum (FWHM) of X-ray diffraction peak (rad.). All the peaks are well defined pointing to the high crystalline nature of the sample. A definite line broadening of the diffraction peaks is an indication that the synthesised materials are in nanometre range. The lattice parameters calculated were also in agreement with the reported values.

Williamson-Hall method

Strain-induced broadening due to crystal imperfection and distortion was calculated using the formula,

$\varepsilon=\frac{\beta_{h k l}}{\tan \theta}$.

The significant property of Eq. (4) is dependent on the diffraction angle $\theta$. Scherrer equation follows a $1 / \cos \theta$ dependency but not $\tan \theta$ as Williamson-Hall method. This elementary difference was that both microstructural cause small crystallite size and micro-strain occur together from the reflection broadening. From Eqs. 4 and 5, it is 


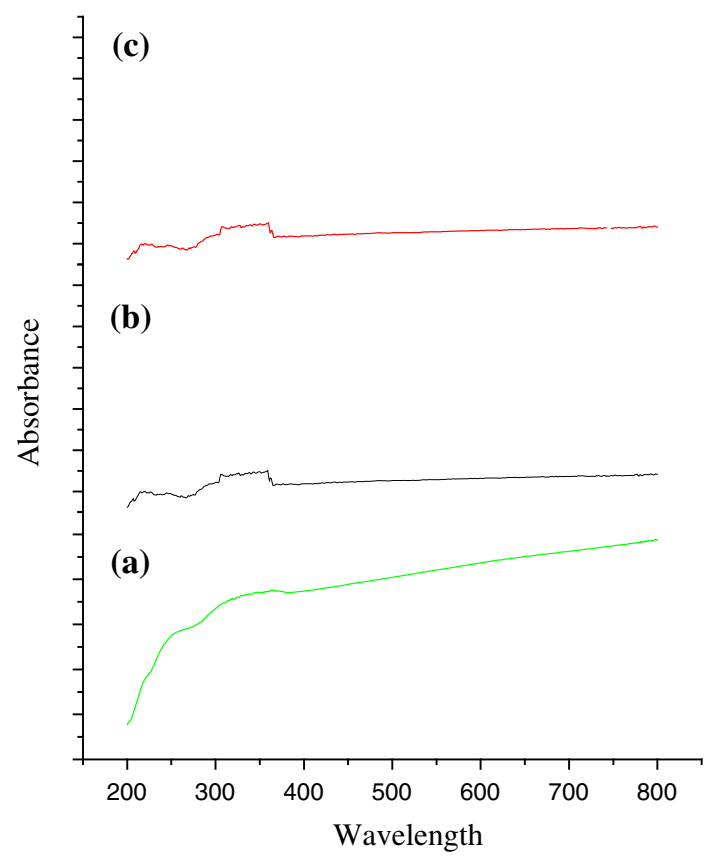

Fig. 4 a UV-Vis result of the $\mathrm{Zn}$ nanohexagons synthesised at optimised condition, b UV-Vis result of the $\mathrm{Zn}$ nanohexagons synthesised at pH 4.2 and $.04 \mathrm{mM}$ concentration, with CTAB, c UVVis result of the $\mathrm{Zn}$ nanohexagons synthesised at $\mathrm{pH} 3.2$ and $0.02 \mathrm{mM}$ concentration, without CTAB

understood that the peak width from crystallite size varies as $\frac{1}{\cos \theta}$ and the strain varies as $\tan \theta$, assuming that the particle size and strain contributions to line broadening are independent to each other and both have a Cauchy-like profile. The observed line breadth is the sum of Eqs. 4 and 5 ,

$\beta_{h k l}=\frac{K \lambda}{D \cos \theta}+4 \varepsilon \sin \theta$

By rearranging the above equation,

$\beta_{h k l} \cos \theta=\frac{K \lambda}{D}+4 \varepsilon \sin \theta$

The above equation is known as Williamson-Hall equation. A plot is drawn with $4 \sin \theta$ along the $x$-axis and $\beta_{h k l} \cos \theta$ along the $y$-axis as shown in figure. From the linear fit to the data, the crystalline size was calculated from the ordinate intercept, and the strain from the slope of the fit. If the point in Williamson-Hall plot is scattered,

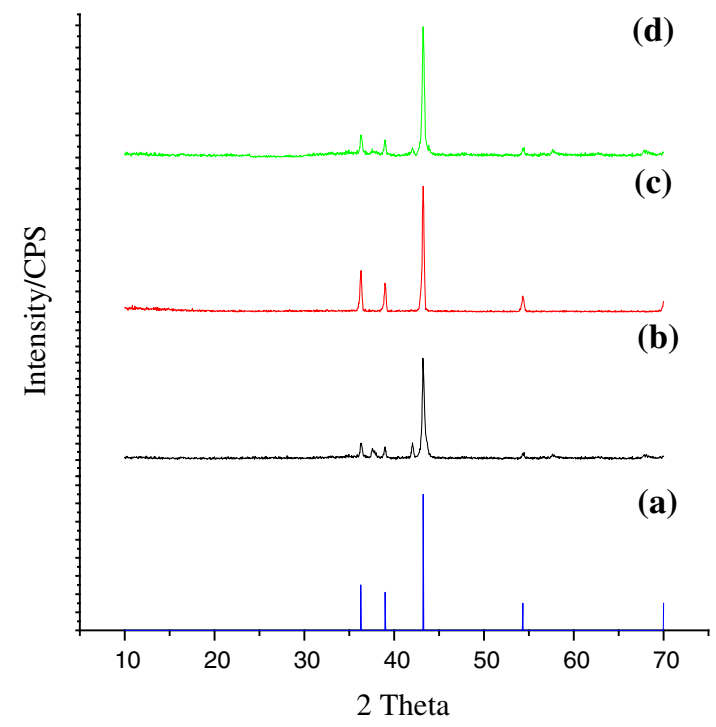

Fig. 5 XRD patterns of the Zn nanohexagons synthesised at different reaction conditions, a standard XRD pattern for Zinc (ICSD No. 064990), b pH 3.2 and $0.02 \mathrm{mM}$ concentration, with CTAB, c pH 3.6 and $0.01 \mathrm{mM}$ concentration, without CTAB, d pH 4.2 and $0.04 \mathrm{mM}$ concentration, with $\mathrm{CTAB}$

that is $\beta_{h k l} \cos \theta$ is not a monotonous function of $4 \sin \theta$ the broadening is termed as anisotropic. The uniform deformation model of the above equation for the Zn NPs is shown in Fig. 6. The results obtained from the Williamson-Hall plot showed that the line broadening was essentially anisotropic. Anisotropic broadening of the diffraction peaks usually arises from the strain field due to dislocation in the sample. The actual particle size taking strain into account is higher than that evaluated using Scherrer equation which does not take strain broadening into account. The geometric parameters of the $\mathrm{Zn}$ nanohexagons synthesised at different reaction conditions are summarised in Table 1.

The texture coefficient has been used to describe the preferred orientation.

$\mathrm{TC}=\frac{I(h k l) / I_{0}(h k l)}{\frac{1}{N} \sum I(h k l) / I_{0}(h k l)} \times 100 \%$

where $I(h k l)_{i}$ is the measured peak intensity of the $(h k l)$ plane for the $i$ th peak, $I_{0}(h k l)_{i}$ is the standard intensity (JCPDS) of the $(h k l)$ plane for the $i$ th peak, and $N$ is the
Table 1 Geometric parameters of the Zn Nps synthesised at different reaction conditions

\begin{tabular}{|c|c|c|c|c|c|c|c|c|}
\hline \multirow[t]{2}{*}{ Sample } & \multirow{2}{*}{$\begin{array}{l}\mathrm{ZnSO}_{4} \\
\text { conc. }(\mathrm{mM})\end{array}$} & \multirow{2}{*}{$\begin{array}{l}\text { CTAB } \\
\text { conc. (mM) }\end{array}$} & \multirow[t]{2}{*}{$\mathrm{pH}$} & \multirow{2}{*}{$\begin{array}{l}\text { Scherrer } \\
\text { method, } \\
D(\mathrm{~nm})\end{array}$} & \multicolumn{2}{|c|}{ Williamson-Hall method } & \multirow{2}{*}{$\begin{array}{l}\text { TEM grain } \\
\text { size, } D(\mathrm{~nm})\end{array}$} & \multirow[t]{2}{*}{$E_{\mathrm{g}}(\mathrm{eV})$} \\
\hline & & & & & $\begin{array}{l}\text { Grain size, } \\
D(\mathrm{~nm})\end{array}$ & $\begin{array}{l}\text { Strain } \\
\varepsilon \times 10^{-4}\end{array}$ & & \\
\hline I & 0.02 & 0.005 & 3.2 & 29 & 33 & 11 & 50 & 3.38 \\
\hline II & 0.01 & - & 3.6 & 34 & 37 & 9 & 67 & 3.47 \\
\hline III & 0.04 & 0.01 & 4.2 & 38 & 42 & 6 & 100 & 3.46 \\
\hline
\end{tabular}



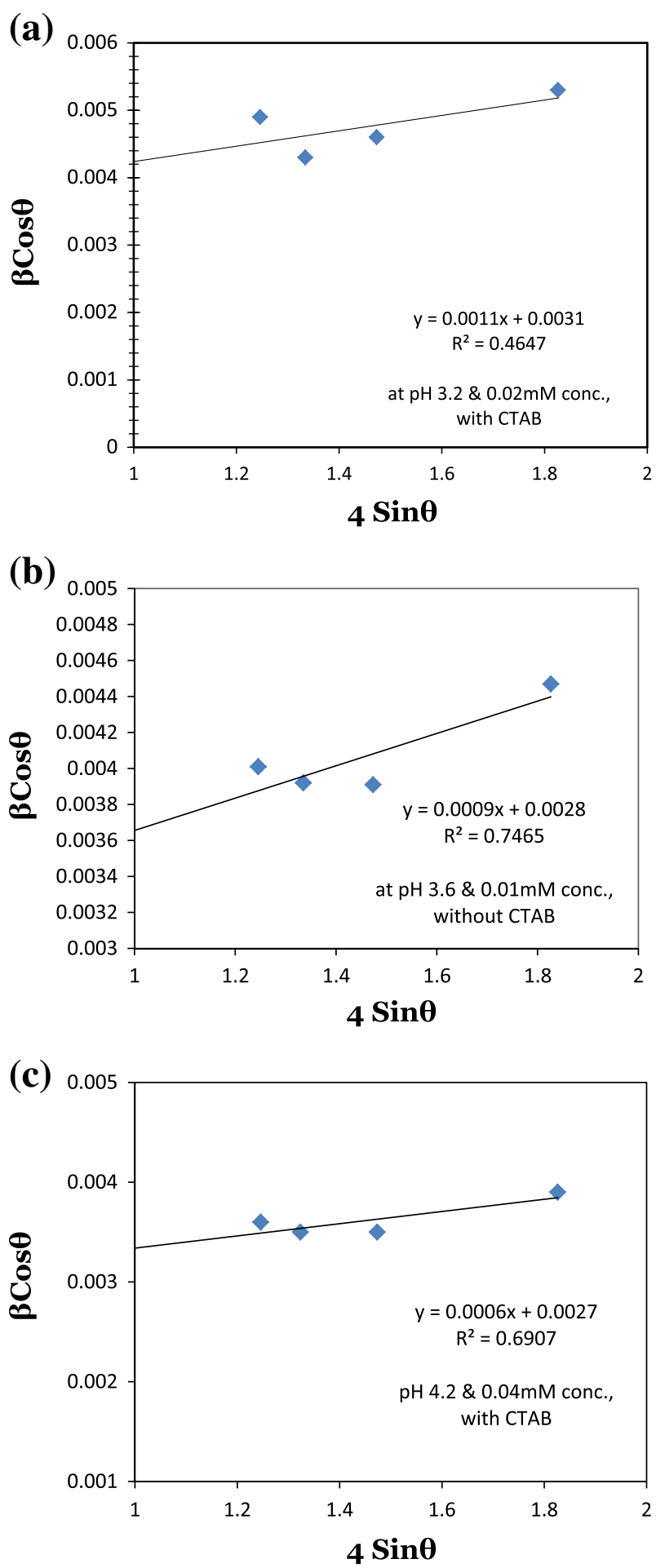

Fig. 6 The W-H analysis of Zn NPs synthesised at different conditions are shown in Fig. $4 \mathrm{a}-\mathrm{c}$. Fit to the data, the strain is extracted from the slope and the crystalline size is extracted from the $y$-intercept of the fit

number of diffraction peaks. The value $\mathrm{TC}(h k l)=1$ represents deposition with randomly oriented crystallites, while deviation of the texture coefficient from unity
Table 2 The texture coefficient for (101) plane for the $\mathrm{Zn}$ nanohexagons synthesised at different reaction parameters

\begin{tabular}{llll}
\hline Sample & FWHM $(101)$ & Grain size $(\mathrm{nm})$ & $\mathrm{TC}_{101}$ \\
\hline I & 0.2891 & 29 & 2.06 \\
II & 0.2415 & 35 & 1.48 \\
III & 0.2145 & 39 & 1.56 \\
\hline
\end{tabular}

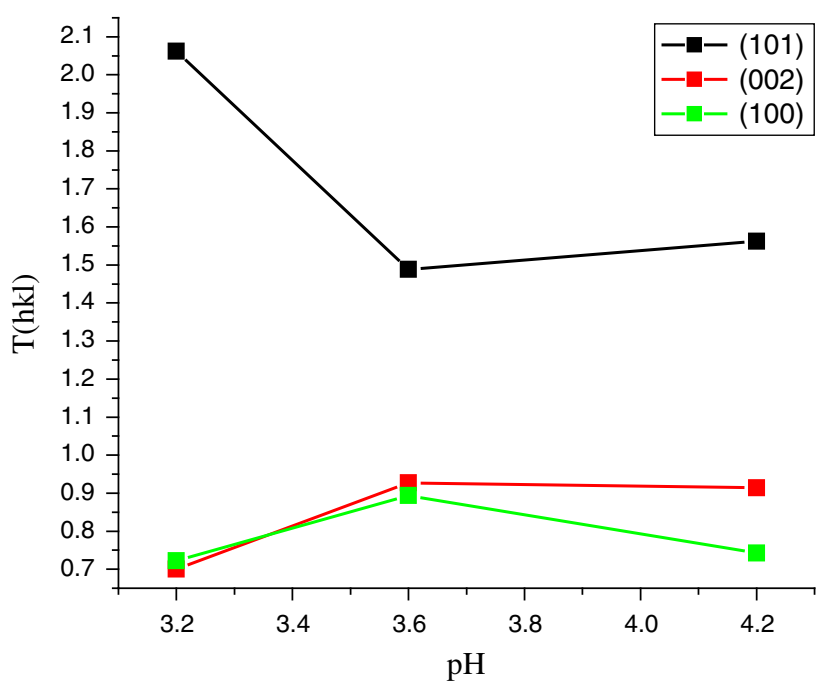

Fig. 7 Texture coefficient $T(h k l)_{i}$ of the Zn NPs deposited at different $\mathrm{pH}$

implies the preferred growth. The variation of TC for the peaks of the Zn NPs lattice is presented in Table 2. The texture coefficient finds the (101) plane as the preferred orientation in the $\mathrm{Zn}$ nanohexagons. Table 2 shows the texture coefficient of the (101) plane for different deposition conditions.

To investigate the influence of the reaction parameter on the preferred orientation, the coefficients $T(h k l)_{i}$ of three intense lines, namely, $T(002), T(100)$, and $T(101)$ were used. Increasing the $\mathrm{pH}$, the intensity of $T(101)$ decreases rapidly and then showed a gradual increase, while the intensity of $T(002)$ increases and then slightly decreases and the intensity of $T(100)$ increases and decreases. The preferred orientation along the (101) plane was exhibited at $\mathrm{pH} 3.2$, as shown in the Fig. 7.

\section{Conclusion}

Zinc nanohexagons were synthesised by electrodeposition method without any nanoparticle stabilisers. This method is a rapid and eco-friendly technique to obtain Zn NPs. SEM/ TEM observations have shown that the morphology of electrodeposited $\mathrm{Zn}$ nanocrystals is found to vary with electrolytic concentration, $\mathrm{pH}$, and additives. TEM 
investigations have shown that the grown nanoparticles are crystalline in nature. Based on the electron microscope images it is concluded that various forms of Zn NPs were formed depending on the reaction conditions. The XRD results indicated the HCP structure of the $\mathrm{Zn} \mathrm{Nps}$ and the line broadening observed in the sample synthesised at optimum condition was due to small crystallite size and lattice strain. Particle size was calculated using DebyeScherrer formula and $\mathrm{W}-\mathrm{H}$ analysis. From the XRD results it was observed that the crystallite size calculated by W-H analysis was higher than that calculated from DebyeScherrer formula. It was also observed that the particle size increased and strain value decreased deviating from the optimum condition. A blue shift of the band edge for the $\mathrm{Zn}$ Nps showed the influence of the reaction conditions. Plasmon absorption of $\mathrm{Zn}$ Nps shows that they are the potential candidates for wide band gap $(3.38 \mathrm{eV})$.

Open Access This article is distributed under the terms of the Creative Commons Attribution License which permits any use, distribution, and reproduction in any medium, provided the original author(s) and the source are credited.

\section{References}

1. Putzbach, W., Ronkainen, N.J.: Sensors 13, 4811-4840 (2013)

2. Chen, J., Miao, Y., He, N., Wu, X., Li, S.: Biotechnol. Adv. 22, 505-518 (2004)

3. Freitas Jr, R.A.: Nanodentistry. J. Am. Dent. Assoc. 131(1559e), 1566e (2000)

4. Li, G., Wang, B., Liy, Y., Tan, T., Song, X., Yan, H.: Appl. Surf. Sci. 255, 3112-3116 (2008)
5. Deiss, E., Holzer, F., Hans, O.: Electrochem. Acta 47, 3995-4010 (2002)

6. Kaur, M., Bhattacharya, S., Roy, M., Deshpande, S.K., Sharma, P., Gupta, S.K., Yakhmi, J.V.: Appl. Phys. A Mater. Sci. Process. 87, 91-96 (2007)

7. Veleva, L., Meraz, E., Acosta, M.: Mater. Corros. 58, 348-352 (2007)

8. Rajendran, S., et al.: Port. Electrochim. Acta 27, 555-564 (2009)

9. Youssef, K.M.S., et al.: Corros. Sci. 46, 51-64 (2004)

10. Astruc, D., Lu, F., Aranzaes, J.R.: Angew. Chem. Int. Ed. 44, 7852-7872 (2005)

11. Singh, S.C., Gopal, R.: Bull. Mater. Sci. 30(3), 291-293 (2007)

12. Kar, S., Ghoshal, T., Chaudhari, S.: Chem. Phys. Lett. 419, 174-178 (2006)

13. Peng, X.S., Zhang, L.D., Meng, G.W., Yuan, X.Y., Lin, Y., Tian, Y.T.: J. Phys. D Appl. Phys. 36, L35-L38 (2003)

14. Wen, X., Fang, Y., Yang, S.: Angew. Chem. Int. Ed. 44, 3562-3565 (2005)

15. Usha, R., et al.: International Proceedings of Chemical, Biological and Environmental Engineering (IPCBEE), vol. 39 (2012)

16. Egorova, E.M.: Russ. J Phys. Chem. A Focus Chem. 84(4), 629-635

17. Kim, S.J., Kim, H.T., Park, S.M.: J. Electrochem. Soc. 151(12), C850-C854 (2004)

18. Wang, J.G., Tian, M.L., Kumar, N., Mallouk, T.E.: Nano Lett. 5(7), 1247-1253 (2005)

19. Wang, S., Zhang, K., et al.: J. Phys. Soc. Jpn. 72(6) 2003

20. Saber, K., Koch, C.C. Fedkiw, P.S.: Mater. Sci. Eng. A341, 174/181 (2003)

21. Tuaweri, T.J., Adigio, E.M., Jombo, P.P.: IJESI.org 2(8), 17-24 (2013)

22. Ramesh Bapu, G.N.K., Devaraj, G., Ayyapparaj, J.: J. Solid State Electro. Chem. 3(1), 48-51 (1998)

23. Shanmugasigamani, S., et al.: Portugaliae Electrochimica Acta 27(6), 725-735 (2009)

24. Youssef, K.M.S., Koch, C.C., Fedkiw, P.S.: J. Electrochem. Soc. 151(2), C103-C111 (2004)

25. Nor, H., et al.: IIUM Eng. J. 12(5) (2011). Special Issue on Science and Ethics in Engineering 